\title{
SOME APPLICATIONS OF THE EULER-JACOBI FORMULA TO DIFFERENTIAL EQUATIONS
}

\author{
ANNA CIMA, ARMENGOL GASULL, AND FRANCESC MAÑOSAS
}

(Communicated by Charles Pugh)

\begin{abstract}
The Euler-Jacobi formula gives an algebraic relation between the critical points of a vector field and their indices. Using this formula we obtain an upper bound for the number of centers that a planar polynomial differential equation can have and study the distribution of the critical points for planar quadratic and cubic differential equations.
\end{abstract}

\section{Statement of Main Results}

In this paper we apply the Euler-Jacobi formula to study two different kinds of problems in the qualitative theory of ordinary differential equations: the number of centers for planar polynomial differential equations and the relationship between the indices of critical points and their distribution.

This formula will be stated in the following section. Here we state the main results in each of the subjects.

A. Centers for planar polynomial differential equations. We denote by $\mathscr{X}_{n, m}$ the set of all polynomial vector fields $X=(P, Q)$ such that the degrees of $P$ and $Q$ are $n$ and $m$, respectively. Without loss of generality we can assume that $n \geq m$. We denote by $E(\cdot)$ the integer part function.

Our aim is to study the number of centers that $X \in \mathscr{X}_{n, m}$ can have. Define

$C_{n, m}=$ maximum number of centers for $X \in \mathscr{Z}_{n, m}$,

$P_{n, m}=$ maximum number of points with index +1 for $X \in \mathscr{X}_{n, m}$,

$N_{n, m}=$ maximum number of points with index -1 for $X \in \mathscr{X}_{n, m}$.

It is obvious that $C_{n, m} \leq P_{n, m}$. The numbers $P_{n, m}$ are studied in [K, CL]. We have that

$$
P_{n, m}= \begin{cases}\frac{(n+1) m}{2} & \text { if } n \equiv m(\bmod 2), \\ \frac{n m}{2} & \text { if } n \neq m(\bmod 2) .\end{cases}
$$

Our main result can be stated as follows:

Received by the editors August 19, 1991.

1991 Mathematics Subject Classification. Primary 34C05, 58F21.

Key words and phrases. Differential equation, critical point, Euler Jacobi formula, center point.

Partially supported by the DGICYT grant number PB90-0695. 
Theorem A. The number $C_{n, n}$ for $n>1$ satisfies

$$
E\left(\frac{n^{2}+1}{2}\right) \leq C_{n, n} \leq P_{n, n}-1=\frac{n^{2}+n}{2}-1 .
$$

We summarize the above results:

Theorem B. The numbers $C_{n, m}$ satisfy

(i) $C_{n, m}=\frac{n m}{2}$ if $n \not \equiv m(\bmod 2)$;

(ii) $E\left(\frac{n m+1}{2}\right) \leq C_{n, m} \leq P_{n, m}=\frac{(n+1) m}{2}$ if $n \neq m, n \equiv m(\bmod 2)$;

(iii) $E\left(\frac{n^{2}+1}{2}\right) \leq C_{n, n} \leq P_{n, n}-1=\frac{n^{2}+n}{2}-1$ if $n=m, n>1$.

Note that for most values of $n$ and $m$ there is a gap between the given upper and lower bounds for $C_{n, m}$. In fact except for the case $n \not \equiv m(\bmod 2)$ the only $C_{n, m}$ known are $C_{0,0}=0, C_{1,1}=1, C_{2,2}=2$, and $C_{3,3}=5$. Among these four cases the first two are obvious. The third one is a classical result on quadratic systems (see [C]), which is proved by studying the vector field on the straight lines joining the critical points.

The proof that $C_{3,3}=5$ and, in general, the proof of Theorem $\mathrm{A}$, needs more powerful tools. This is where we need the algebraic constraint given by the Euler-Jacobi formula. In fact the proof of Theorem A shows that for $X \in \mathscr{X}_{n, n}$, the vector field has at most $P_{n, n}-1$ critical points with index +1 on the curve $\operatorname{div} X=\partial P / \partial x+\partial Q / \partial y=0$. Hence, a result similar to Theorem A, with weak focus instead of centers, holds.

On the other hand the lower bounds given in Theorems A and B are realized by Hamiltonian vector fields, that is, for vector fields $X=(\partial H / \partial x,-\partial H / \partial y)$ $\in \mathscr{X}_{n, m}$, where $H$ is a polynomial of degree $n+1$. In fact, it is proved in [CGM] that for these Hamiltonian vector fields, the lower bound $E\left(\frac{n m+1}{2}\right)$ is actually the maximum number of centers that a planar polynomial Hamiltonian differential equation in $X_{n, m}$ can have. Conti has already observed that $C_{n, n} \geq$ $E\left(\frac{n^{2}+1}{2}\right)$ and conjectured that the equality holds.

Finally we stress that the easiest problem that remains to be solved is to find $C_{4,4}$. By Theorem A, $8 \leq C_{4,4} \leq 9$.

B. Indices of critical points and their distribution. For quadratic systems, i.e., $X \in \mathscr{X}_{2,2}$, we have the following:

Berlinskiir's Theorem (see [B]). Suppose that $X \in \mathscr{X}_{2,2}$ has four real critical points. If the quadrilateral with vertices at these points is convex then two opposite critical points are saddles (index -1) and the other two are antisaddles (nodes, foci or centers, index +1$)$. But if the quadrilateral is not convex then either the three exterior vertices are saddles and the interior vertex an antisaddle, or vice-versa.

A proof of this theorem using coordinates can be bound in [C], and a different one, using pencils of conics in [S].

For $X \in \mathscr{X}_{n, m}$ we denote by $A_{X}=A$ the set $\left\{p \in \mathbb{R}^{2}: X(p)=0\right\}$ of critical points of $X$. Given a subset of $\mathbb{R}^{2}, B$, we denote by $\widehat{B}$ its convex hull, by $\partial B$ its boundary, and by $\# B$ its cardinal.

Set $A_{0}=A$, and for $i \geq 1$ set $A_{i}=A_{i-1}-\left(A_{i-1} \cap \partial \widehat{A_{i-1}}\right)$; there is a last integer $p$ for which $A_{p+1}=\varnothing$. 


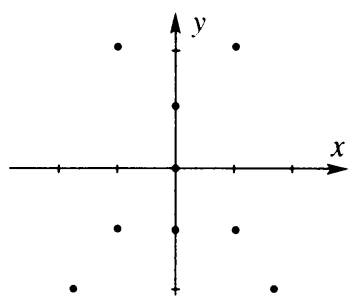

FIGURE 1. Example of configuration $(4 ; 4 ; 1)$

We will say that $A$ has configuration $\left(K_{0} ; K_{1} ; \cdots ; K_{p}\right)$ where $K_{i}$ is the natural positive number defined by

$$
K_{i}=\#\left(A_{i} \cap \partial \widehat{A_{i}}\right) .
$$

Observe that this is not the definition of configuration used in [Ko]. For example, if we take the system considered in [Ko], $\dot{x}=-x\left(1+\frac{1}{2} y-\frac{3}{4} y^{2}+x^{2}\right)$, $\dot{y}=(y+1)\left(y^{2}-y-2 x^{2}\right)$, then $X \in \mathscr{Z}_{3,3}$ and $A=\left\{(0,0),(0, \pm 1),\left( \pm \frac{1}{2},-1\right)\right.$, $( \pm 1,2),( \pm \sqrt{3},-2)\}$. Using our notation its configuration is $(4 ; 4 ; 1)$, see Figure 1 .

We will say that $A$ has configuration $\left(K_{0} ; K_{1} ; \cdots ; K_{r} ; *\right)$ if we do not specify for the values of $K_{i}$ for $i$ between $r+1$ and $p$.

We are also interested in the study of the indices at the critical points of $X$. We will say that critical points of $X$, which belong to $A_{i} \cap \partial \widehat{A_{i}}$, are on the $i t h$ level.

If we assume that $X \in \mathscr{Z}_{n, m}$ is such that $\# A_{X}=n m$, then the indices are \pm 1 , and in this case we substitute the number $K_{i}$ corresponding to the $i$ th level by $\left(n_{i}^{1}+, n_{i}^{2}-, n_{i}^{3}+, \ldots, n_{i}^{m_{i}}-\right)$ where $n_{i}^{j}$ are positive integers such that $\sum_{j} n_{i}^{j}=K_{i}$. When $A_{i} \cap \partial \widehat{A_{i}}$ is a polygon, these numbers take into account the number of consecutive points with positive and negative indices, viewing the $i$ th level oriented counterclockwise; $n_{i}^{1}$ corresponds to the string with largest number of consecutive points with positive indices. If there are several strings with the same number of points we choose one such that the next string (that has points with negative indices) is as large as possible, and so on. When $A_{i} \cap \partial \widehat{A_{i}}$ is a segment, the numbers $n_{i}^{j}$ take into account the number of consecutive points with positive and negative indices, beginning at one of its endpoints.

The example in Figure 1 has configuration $(4+;+, 3-;+)$ where the number 1 is omitted.

In this notation, Berlinskii's Theorem can be stated as follows:

Berlinskii's Theorem. Assume that $X \in \mathscr{Z}_{2,2}$ and $\# A_{X}=4$. Then

(1) $\sum_{a \in A} i_{X}(a)=0$ if and only if the configuration of the set $A$ is (4) = $(+,-,+,-)$

(2) $\left|\sum_{a \in A} i_{X}(a)\right|=2$ if and only if the configuration of $A$ is $(3 ; 1)$. In this case it can be either $(3+;-)$ or $(3-;+)$.

In the last section we will give a proof of the above result, different from the proofs that appear in $[\mathrm{C}, \mathrm{S}]$. We will also prove the following generalizations: 
Theorem C. (a) Assume that $X \in \mathscr{X}_{3,2}$ and $\# A_{X}=6$. Then $\sum_{a \in A} i_{X}(a)=0$ and only the following configurations are possible:

(i) $(6)=(+,-,+,-,+,-)$,

(ii) $(4 ; 2)=(2+, 2-;+,-)$,

(iii) $(3 ; 3)=(2+,-;+, 2-)$ or $(+, 2-; 2+,-)$.

Furthermore there exist $X \in \mathscr{Z}_{3,2}$ with the above configurations.

(b) Assume that $X \in \mathscr{X}_{3,3}$ and \# $A_{X}=9$. Then $\left|\sum_{a \in A} i_{X}(a)\right| \in\{1,3\}$.

(b1) If $\left|\sum_{a \in A} i_{X}(a)\right|=3$ only the following configurations are possible:

(i) $(5 ; 3 ; 1)=(5+; 3-;+)$ or $(5-; 3+;-)$,

(ii) $(4 ; *)=(4+; *)$ or $(4-; *)$,

(iii) $(3 ; *)=(3+; *)$ or $(3-; *)$.

Furthermore there exist examples of configurations: $(5 ; 3 ; 1),(4 ; 4 ; 1)$, $(4 ; 3 ; 2)$, and $(3 ; 6)$.

(b2) If $\left|\sum_{a \in A} i_{X}(a)\right|=1$ there exist examples of the configurations: $(8 ; 1)$, $(7 ; 2) ;(6 ; 3) ;(5 ; 4) ;(5 ; 3 ; 1) ;(4 ; 5),(4 ; 4 ; 1),(4 ; 3 ; 2) ;(3 ; 3 ; 3)$.

Figures 2, 3, and 4 show examples of most of the configurations considered. In these figures we plot the points of $P(x, y)=0$ with continuous trace and the points of $Q(x, y)=0$ with discontinuous trace.

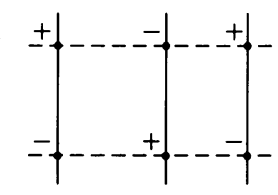

(6) $=(+,-,+,-,+,-)$

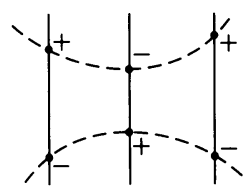

$(4 ; 2)=(2+, 2-;+,-)$

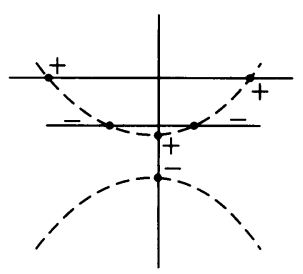

$(3 ; 3)=(2+,-;+, 2-)$

FigURE 2. Configurations for $X \in \mathscr{Z}_{3,2}$

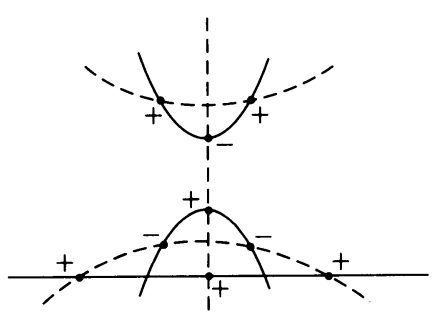

$(5 ; 3 ; 1)=(5+; 3-;+)$

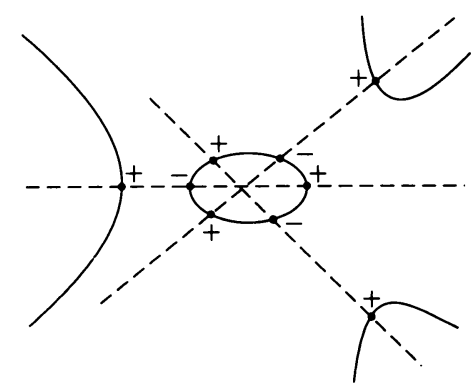

$(3 ; 6)=(3+;+,-,+,-,+,-)$

Figure 3. $(5 ; 3 ; 1)$ and $(3 ; 6)$ configurations for $X \in$ $\mathscr{Z}_{3,3}$ with $\sum_{a \in A} i_{X}(a)=3$ 


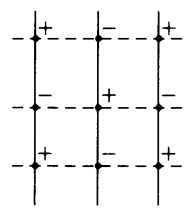

$(8 ; 1)$

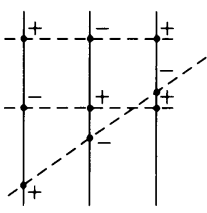

$(7 ; 2)$

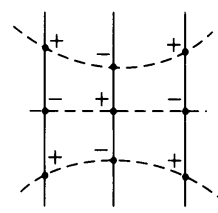

$(6 ; 3)$

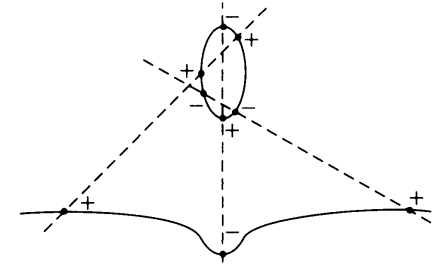

$(5 ; 4)$

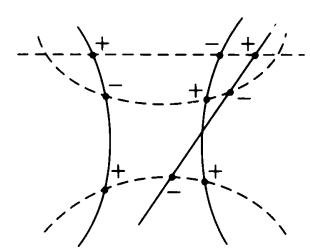

$(5 ; 3 ; 1)$

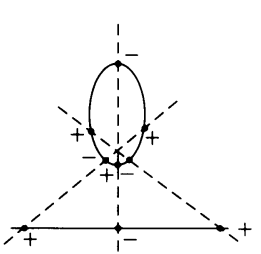

$(4 ; 5)$

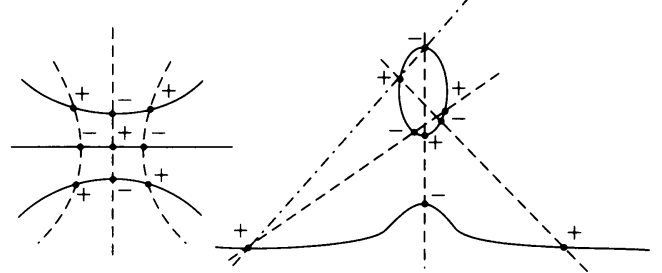

$(4 ; 4 ; 1)$

$(4 ; 3 ; 2)$

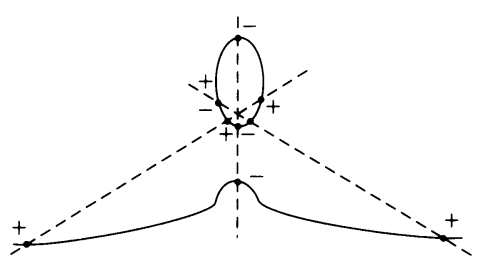

$(3 ; 3 ; 3)$

Figure 4. Configurations for $X \in \mathscr{X}_{3,3}$ with $\sum_{a \in A} i_{X}(a)=1$

We remark that there are some problems that remain to be solved in connection with the problem of determining the possible configurations of $A$ for $X \in \mathscr{X}_{3,3}$. One of the more interesting is whether it is possible to have (9) with $\left|\sum_{a \in A} i_{x}(a)\right|=1$ occurring as a configuration. Note that we have not studied all possible combinations of indices in the $i$ th levels. For instance, the $(6 ; 3)$ configuration with $\sum_{a \in A} i_{x}(a)=1$ can have at least the two different distributions of indices $(2+,-, 2+,-;-,+,-)$ and $(3+,-,+,-;+, 2-)$ as depicted in Figure 5.

Some partial results can also be proved for $n$-dimensional vector fields by using the same techniques. We have

Theorem D. Let $X=\left(P_{1}, P_{2}, \ldots, P_{n}\right)$ be a polynomial vector field on $\mathbb{R}^{n}$, with $\operatorname{deg} P_{1}=\operatorname{deg} P_{2}=2$ and $\operatorname{deg} P_{i}=1$ for $i \geq 3$. Then if $\# A_{X}=4$, the four critical points are in a plane of $\mathbb{R}^{n}$ (not necessarily invariant), and on this plane Berlinskii's Theorem holds. 


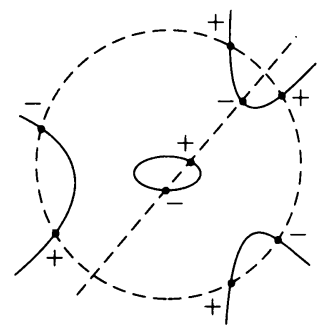

$(2+,-, 2+,-;-,+,-)$

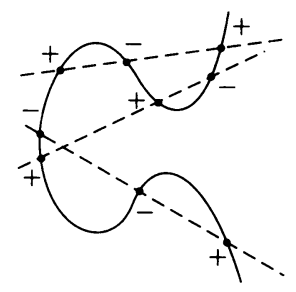

$(3+,-,+,-;+, 2-)$

Figure 5. Different examples of $(6 ; 3)$ configurations

We omit the proof because it is essentially the same as our proof of Berlinskiî's Theorem.

\section{Preliminary Results}

Remember that for a simple critical point $p$ of a differential equation in $\mathbb{R}^{2}$, $(\dot{x}, \dot{y})=X(x, y)$ where $X=(P, Q)$,

$$
J(p)=\operatorname{det}\left(\frac{\partial(P, Q)}{\partial(x, y)}\right)
$$

does not vanish and $\operatorname{sgn}(J(p))$ is the index of $X$ at $p$. As usual we will denote it by $i_{X}(p)=i(p)$.

The Euler-Jacobi formula gives a nonobvious relation between the critical points of a polynomial differential equation and their indices, assuming that all of them are simple and lie in the complex plane. A proof of it can be found in [AVG] or in [GH, p. 671].

First we enunciate a corollary of Bezout's Theorem.

Proposition 2.1. Consider two algebraic curves $P=0, Q=0$ with degrees $n$ and $m$ respectively. Then the number of nondegenerate solutions of the system $P=Q=0$ is finite and less or equal than $\mathrm{nm}$.

In fact Bezout's Theorem ensures that in the complex projective plane (and taking into account multiplicities), if this number is finite, it is exactly $\mathrm{nm}$.

The Euler-Jacobi formula deals with the case in which all these solutions are simple and lie in the finite part of the complex projective space. Now we can enunciate this formula for the two-dimensional case.

The Euler-Jacobi formula (EJ formula). Consider a system of two real polynomial equations of degrees $n, m$ in 2 complex unknowns

$$
P(x, y)=Q(x, y)=0 .
$$

If we assume that the set of roots of the system $A$ contains exactly $\mathrm{nm}$ elements, we have that $J=\operatorname{deg}\left(\frac{\partial(P, Q)}{\partial(x, y)}\right)$ does not vanish on $A$. Then for every polynomial $R$ of degree less than $n+m-2$ we have

$$
\sum_{a \in A} \frac{R(a)}{J(a)}=0 \text {. }
$$


Some results that relate critical points of a polynomial differential equation with their indices can be found in [K], see also [CL].

Theorem 2.2. Let $X=(P, Q)$ be a polynomial vector field on $\mathbb{R}^{2}$ with finitely many critical points. Then

(i) $\left|\sum_{a \in A} i_{x}(a)\right| \leq \min (n, m)$; furthermore, if \# $A=n m$ then we have $\left|\sum_{a \in A} i x(a)\right| \equiv n m(\bmod 2)$.

(ii) If $n \not \equiv m(\bmod 2)$ and $\#\left\{a \in \mathbb{C}^{2}: P(a)=Q(a)=0\right\}=n m$ then $\sum_{a \in A} i_{x}(a)=0$

(iii) $\sum_{a \in A}\left|i_{x}(a)\right| \leq n m$.

(iv) If $n \equiv m(\bmod 2)$, assuming that $m \leq n$, then $P_{n, m}, N_{n, m} \leq$ $(m+n m) / 2$.

(v) If $n \not \equiv m(\bmod 2)$ then $P_{n, m}, N_{n, m} \leq n m / 2$.

From the work of Poincare there is a geometrical way of knowing the indices at the critical points.

Proposition 2.3. Let $X=(P, Q)$ be an analytical vector field defined on $\mathbb{R}^{2}$. Let $p, q$ be two consecutive critical points on a real connected component of $P=0$ such that $J(p) J(q) \neq 0$ and $P=0$ has no multiple points between $p$ and $q$. Then $J(p) J(q)<0$.

This proposition is used systematically and without being explicitly mentioned in the construction of examples made in $\S 3$. A proof of it can be found in [CL].

We will use (sometimes also without being explicitly mentioned) this wellknown technical lemma (see [Ch]).

Lemma 2.4. Let $X=(P, Q)$ be a polynomial vector field with $\max (\operatorname{deg} P, \operatorname{deg} Q)$ $=n$. If $X$ has $n$ critical points on a straight line $L(x, y)=0$, this line is an isocline. If $X$ has $n+1$ critical points on $L(x, y)=0$ then this line is full of critical points.

Finally we utilize Newton's classification (see $[\mathrm{BK}]$ ) to draw the sets $P=0$ and $Q=0$, when $P$ and $Q$ are cubics. Following this classification a cubic that contains no straight lines can be represented, in a suitable cartesian coordinate, in one of the 4 normal forms:

$$
\begin{array}{rlrl}
\text { I: } & x y^{2}+e x y & =P_{3}(x)=a x^{3}+b x^{2}+c x+d, \\
\text { II: } & & x y & =P_{3}(x), \\
& \text { III: } & y^{2} & =P_{3}(x), \\
\text { IV }: & & y & =P_{3}(x) .
\end{array}
$$

\section{Proofs of Theorems A AND B}

The lower bound for Theorems A and B is achieved by the following example.

Example 3.1. The Hamiltonian system

$$
\begin{aligned}
\dot{x} & =y(y-1) \cdots(y-(n-1)), \\
\dot{y} & =(-1)^{m-n+1} x(x-1) \cdots(x-(m-1)),
\end{aligned}
$$

has exactly $E\left(\frac{n m+1}{2}\right)$ centers. 
Proof. Clearly this system has $n m$ critical points. For all $n$ and $m$ the determinant of the linear part at $(0,0)$ is positive, so $\operatorname{ind}(0,0)=+1$. Since the indices on each straight line $x-i=0$ for $i=0, \ldots, m-1$ and $y-j=0$ for $j=0, \ldots, n-1$ are alternated, we obtain $E\left(\frac{n m+1}{2}\right)$ points with index +1 . Note that all simple critical points with positive index for a Hamiltonian system are centers; so we obtain the desired result.

An easy upper bound for $C_{n, m}$ is given by the number of critical points of index +1 , see [CL, Theorem 1.3]. We state it in the next lemma.

Lemma 3.2. Let $X$ be in $\mathscr{X}_{n, m}$. Then $C_{n, m} \leq P_{n, m}$ where

$$
P_{n, m}= \begin{cases}\frac{(n+1) m}{2} & \text { if } n \equiv m(\bmod 2), \\ \frac{n m}{2}=E\left(\frac{n m+1}{2}\right) & \text { if } n \neq \equiv m(\bmod 2) .\end{cases}
$$

From Example 3.1 and Lemma 3.2, Theorem B(i) and (ii) follow. To finish the proof, therefore, it only remains to study the case $\mathscr{X}_{n, n}$. For this case we have that

$$
E\left(\frac{n^{2}+1}{2}\right) \leq C_{n, n} \leq \frac{n^{2}+n}{2},
$$

so we have to improve the upper bound, substituting it for $P_{n, n}-1$.

Assuming that $C_{n, n}=P_{n, n}=\left(n^{2}+n\right) / 2$ we will get a contradiction. We will need some preliminary lemmas and will use the following notation: for $R_{1}, \ldots, R_{k} \in R[x, y]$ we denote by $V\left(R_{1}, \ldots, R_{k}\right)$ the set of common real zeros of all of them. When there is no confusion, given $X=(P, Q)$, we will denote by $A=V(P, Q)$ and by $A^{+}$(resp. $A^{-}$) the set of critical points of $X$ that have positive (resp. negative) index.

Lemma 3.3. Let $X=(P, Q) \in \mathscr{X}_{n, n}$ be a vector field with $P_{n, n}$ critical points with index +1 . Then $X$ has $n^{2}$ critical points and all of them are simple.

Proof. Let $p_{1}, p_{2}, \ldots, p_{\left(n^{2}+n\right) / 2}$ be the critical points of $X$ with index +1 , and let $q_{1}, q_{2}, \ldots, q_{r}$ be the other real critical points of $X$. We claim that $r=\left(n^{2}-n\right) / 2$.

Since the sum of the indices (resp. the absolute values of the indices) of $X$ at all its critical points is bounded by $n$ (resp. $n^{2}$ ), we have that $\sum_{j=1}^{r} i_{X}\left(q_{j}\right)+$ $\left(n^{2}+n\right) / 2 \leq n$ (resp. $\left.\sum\left|i_{X}\left(q_{j}\right)\right|+\left(n^{2}+n\right) / 2 \leq n^{2}\right)$, and it can be easily deduced that

$$
\left|\sum_{j=1}^{r} i_{X}\left(q_{j}\right)\right|=\frac{n^{2}-n}{2}
$$

Let $\mu\left(q_{j}\right)$ be the multiplicity of $X$ at $q_{j}$. From [CL, Lemma 1.4] we have that $\left|i_{X}\left(q_{j}\right)\right| \leq \sqrt{\mu\left(q_{j}\right)} \leq \mu\left(q_{j}\right)$ for each $j \in\{1,2, \ldots, r\}$. Applying Bezout's Theorem we obtain

$$
\frac{n^{2}+n}{2}+\sum_{j=1}^{r} \mu\left(q_{j}\right) \leq \sum_{i=1}^{\left(n^{2}+n\right) / 2} \mu\left(p_{i}\right)+\sum_{j=1}^{r} \mu\left(q_{j}\right) \leq n^{2} .
$$


From (1) and (2) we have that

$$
\frac{n^{2}-n}{2}=\left|\sum_{j=1}^{r} i\left(q_{j}\right)\right| \leq \sum_{j=1}^{r}\left|i\left(q_{j}\right)\right| \leq \sum_{j=1}^{r} \sqrt{\mu\left(q_{j}\right)} \leq \sum_{j=1}^{r} \mu\left(q_{j}\right) \leq \frac{n^{2}-n}{2}
$$

i.e., all the inequalities are in fact equalities. In particular, $\sum_{j=1}^{r} \sqrt{\mu\left(q_{j}\right)}=$ $\sum_{j=1}^{r} \mu\left(q_{j}\right)$. Since $\mu\left(q_{j}\right) \geq 1$, we have that $\mu\left(q_{j}\right)=1$ for all $j \in\{1,2, \ldots, r\}$ and the equality $\sum_{j=1}^{r} \mu\left(q_{j}\right)=\left(n^{2}-n\right) / 2$ implies that $r=\left(n^{2}-n\right) / 2$.

From the claim it is clear that $X$ has $n^{2}$ critical points and in this case it is known that all of them are simple.

Lemma 3.4. Let $X=(P, Q) \in \mathscr{X}_{n, n}$ with $\#(V(P, Q))=n^{2}$, and let $R$ be a polynomial with degree less than $n$. Then $V(P, Q) \not \subset V(R)$.

Proof. Suppose that $V(P, Q) \subset V(R)$. Then $\#(V(Q, R)) \geq n^{2}$ and from Bezout's Theorem it follows that $Q$ and $R$ have a common factor. Let $C$ denote a maximal common factor of $R$ and $Q$ and assume $\operatorname{deg}(C)=k<n$. Then we can write $R=C R_{1}$ and $Q=C Q_{1}$, with $\operatorname{deg} Q_{1}=n-k$ and $\operatorname{deg} R_{1}<$ $n-k$. Since $P$ and $Q$ have maximal intersection we get $\#(V(C, P))=n k$ and $\#\left(V\left(Q_{1}, P\right)\right)=(n-k) n$. Therefore $\#\left(V\left(R_{1}, Q_{1}\right)\right)>(n-k) n$, because $A \subset V(R)$. Also from Bezout's Theorem we get that $R_{1}$ and $Q_{1}$ have a common factor. This gives a contradiction with the fact that $C$ is a maximal common factor of $R$ and $Q$.

In the following two lemmas we assume that $n>2$.

Lemma 3.5. If $X \in \mathscr{X}_{n, n}$ has $n(n+1) / 2$ critical points with positive index, the corresponding $n(n-1) / 2$ critical points with negative index cannot belong to any $V(R)$ for $R$ with $\operatorname{deg} R \leq n-2$.

Proof. Assume that $X$ has $n(n+1) / 2$ critical points with positive index and $n(n-1) / 2$ critical points with negative index, and suppose that $A^{-} \subset V(R)$ with $\operatorname{deg}(R) \leq n-2$. Then $\operatorname{deg}\left(R^{2}\right) \leq 2 n-4<2 n-2$ and we can apply the Euler-Jacobi formula to $R^{2}$. We get

$$
\sum_{x \in A} \frac{R^{2}(x)}{J(x)}=\sum_{x \in A^{-}} \frac{R^{2}(x)}{J(x)}+\sum_{x \in A^{+}} \frac{R^{2}(x)}{J(x)}=\sum_{x \in A^{+}} \frac{R^{2}(x)}{J(x)}=0 .
$$

Since $J(x)>0$ for all $x \in A^{+}$, it follows that $R(x)=0$ for all $x \in A^{+}$. Thus, we obtain $A \subset V(R)$ in contradiction with Lemma 3.4.

Lemma 3.6. For every set $B$ of $n(n-1) / 2-1$ points of $\mathbf{R}^{2}$ there exists a polynomial $R \in \mathbf{R}[x, y]$ with $\operatorname{deg}(R)=n-2$ and $B \subset V(R)$.

Proof. Since given a set $B$ of $k(k+3) / 2$ points in $\mathbf{R}^{2}$ there always exists a polynomial $R$ with degree $k$ and $B \subset V(R)$ (see [F]), it suffices to show that

which holds for all $n \in \mathbf{Z}$.

$$
\frac{n(n-1)}{2}-1 \leq \frac{1}{2}(n+1)(n-2) \text {, }
$$

Proposition 3.7. If $X$ has $n(n+1) / 2$ critical points with positive index then they cannot belong to any $V(R)$ for $R$ with $\operatorname{deg}(R) \leq n-1$.

Proof. Let $R \in \mathbf{R}[x, y]$ with $\operatorname{deg}(R) \leq n-1$, and suppose that $A^{+} \subset V(R)$. Since $\#\left(A^{+}\right)=n(n+1) / 2$, we have by Lemma 3.3 that $\#\left(A^{-}\right)=n(n-1) / 2$. 
For all $x \in A^{-}$let $T_{x}$ be the polynomial given in the above lemma such that $\left(A^{-} \backslash\{x\}\right) \subset V\left(T_{x}\right)$ and $\operatorname{deg}\left(T_{x}\right)=n-2$. Then $\operatorname{deg}\left(R T_{x}\right)=2 n-3<2 n-2$, and we can apply the Euler-Jacobi formula to $R T_{x}$ :

$$
0=\sum_{y \in A} \frac{T_{x}(y) R(y)}{J(y)}=\frac{T_{x}(x) R(x)}{J(x)} .
$$

Hence, $R(x) T_{x}(x)=0$. From Lemma 3.5, $T_{x}(x) \neq 0$ and we obtain $x \in$ $V(R)$. Since this argument is valid for each $x \in A^{-}$we obtain $A \subset V(R)$ in contradiction with Lemma 3.4.

Finally we will need the following theorem, which is proved in [CGM].

Theorem 3.8. Let $X$ be a Hamiltonian vector field in $\mathscr{X}_{n, m}$. Then the maximum number of centers of $X$ is $E\left(\frac{n m+1}{2}\right)$.

Proof of Theorem A. Now suppose $C_{n, n}=P_{n, n}=\left(n^{2}+n\right) / 2$ and we will derive a contradiction. If $n=2$ the assertion of Theorem $\mathrm{A}$ is well known. Assume $n>2$. Then $A^{+} \subset V(\operatorname{div} X)$. If $\operatorname{div} X \equiv 0$ then the theorem follows from Theorem 3.8. If $\operatorname{div} X \not \equiv 0$ then $\operatorname{deg}(\operatorname{div} X) \leq n-1$ and Theorem A follows from Proposition 3.7.

\section{Generalizations of Berlinskil's Theorem}

The new proof of Berlinskii's Theorem that we present gives the key ideas that we will apply in the proof of Theorem C.

First of all we remark that if a configuration exists for some $X \in \mathscr{X}_{n, m}$ with \# $A_{X}=n m$ it is possible to construct the same configuration but interchanging points with index 1 with points with index -1 . It is enough to take $Y=$ $\left(-X_{1}, X_{2}\right)$ instead of $X=\left(X_{1}, X_{2}\right)$. So in all cases we will restrict ourselves to systems such that $\sum_{a \in A} i_{x}(a) \geq 0$.

In all proofs we will denote by $\left\{P_{1}, \ldots, P_{n m}\right\}$ the points of $A$ if we have no information about their indices, by $\left\{P_{1}^{+}, \ldots, P_{k}^{+}\right\}$the set of points in $A$ with positive index, and by $\left\{P_{1}^{-}, \ldots, P_{l}^{-}\right\}$the set of points in $A$ with negative index. Also we will put $L_{i, j}^{v w}$ for the straight line $L_{i, j}^{v w}(x, y)=0$ through the points $P_{i}^{v}$ and $P_{j}^{w}$ where $v, w \in\{+,-, \varnothing\}$ and $i \in\{1, \ldots, k\}, j \in$ $\{1, \ldots, l\}$. Finally we denote by $L_{i}^{v}$ any straight line through a point $P_{i}^{v} \in \partial \widehat{A}$ such that for all $q \in A, L_{i}^{v}(A) \geq 0$, and it is zero only at $q$.

New proof of Berlinskii's Theorem. We have that $X \in \mathscr{X}_{2,2}$ and \# $A_{X}=4$.

Assume that $\sum_{a \in A} i_{x}(a)=0$. If we apply the EJ formula to $L=L_{1,2}^{++}$we have that

$$
\frac{L\left(P_{1}^{-}\right)}{J\left(P_{1}^{-}\right)}+\frac{L\left(P_{2}^{-}\right)}{J\left(P_{2}^{-}\right)}=0 .
$$

Since $\operatorname{sgn}\left(J\left(P_{1}^{-}\right)\right)=\operatorname{sgn}\left(J\left(P_{2}^{-}\right)\right)=$minus, we have that $L\left(P_{1}^{-}\right) L\left(P_{2}^{-}\right)<0$ (note that $\left|L\left(P_{1}^{-1}\right)\right|+\left|L\left(P_{2}^{-}\right)\right|$must be different from zero because if $X \in$ $\mathscr{X}_{2,2}$ and $\# A_{X}<\infty$ by Lemma 2.4 it is impossible that 3 critical points are aligned). Hence $P_{1}^{-}$and $P_{2}^{-}$lie on opposite sides of $L$. Then $A$ must have configuration $(4)=(+,-,+,-)$.

Now assume that the configuration of $A$ is (4). Also we put the subscripts of $P_{i}$ in such a way that $P_{1}, P_{2}, P_{3}, P_{4}$ are ordered counterclockwise in $\partial \hat{A}$. Set 
$L_{i}=L_{i, i+1}$ and consider the subscripts in $\mathbb{Z} / 4 \mathbb{Z}$. Again using the EJ formula we have

$$
\frac{L_{i}\left(P_{i+2}\right)}{J\left(P_{i+2}\right)}+\frac{L_{i}\left(P_{i+3}\right)}{J\left(P_{i+3}\right)}=0 .
$$

Since $\operatorname{sgn} L_{i}\left(P_{i+2}\right)=\operatorname{sgn} L_{i}\left(P_{i+3}\right)$, we have that $\operatorname{sgn} J\left(P_{i+2}\right) \neq \operatorname{sgn} J\left(P_{i+3}\right)$. Hence configuration (4) must be (+, -, +, -) and, of course, $\sum_{a \in A} i_{x}(a)=0$.

Assume that $\sum_{a \in A} i_{x}(a)=2$. Take the three straight lines $L_{1}=L_{1,2}^{++}$, $L_{2}=L_{1,3}^{++}$, and $L_{3}=L_{2,3}^{++}$. By the EJ formula for $i=1,2,3$ we have

$$
\frac{L_{i}\left(P_{i}^{+}\right)}{J\left(P_{i}^{+}\right)}+\frac{L_{i}\left(P_{1}^{-}\right)}{J\left(P_{1}^{-}\right)}=0 .
$$

Since $\operatorname{sgn} J\left(P_{i}^{+}\right) J\left(P_{1}^{-}\right)<0$, we have that $P_{i}^{+}$and $P_{1}^{-}$lies on the same side of $L_{i}=0$. Hence $P_{1}^{-}$must be in the interior of the triangle with vertices $P_{i}^{+}$. Then the configuration of $A$ is $(3 ; 1)=(3+;-)$.

Consider now that the configuration is $(3 ; 1)$. Using exactly the same arguments that in the proof of the case in which the configuration is (4) but only for the points $\left\{P_{1}, P_{2}, P_{3}\right\}$ that are in $\partial \hat{A}$ we have that $(3 ; 1)=(3+;-)$, so $\sum_{a \in A} i_{x}(a)=2$.

Proof of Theorem C. The proof of this theorem will follow in two different steps. In the first one we will give proofs of which configurations are possible and which are not possible. In the second one we will give examples of possible configurations.

Step 1. (a) From Theorem 2.2(ii) we see that if $X \in \mathscr{Z}_{3,2}$ then $\sum_{a \in A} i_{x}(a)=$ 0 . Assume that the configuration is (6) and that the subscripts of the points of $A$ are in such a way that $P_{1}, P_{2}, P_{3}, P_{4}, P_{5}, P_{6}$ are ordered in $\partial \widehat{A}$ in counterclockwise sense. Also we consider the subscripts in $\mathbb{Z} / 6 \mathbb{Z}$. Take $Q_{i}(x, y)=$ $L_{i, i+1}(x, y) L_{i+2, i+3}(x, y)$. The EJ formula applied to $Q_{i}$ gives

$$
\frac{Q_{i}\left(P_{i+4}\right)}{J\left(P_{i+4}\right)}+\frac{Q_{i}\left(P_{i+5}\right)}{J\left(P_{i+5}\right)}=0,
$$

so $J\left(P_{i+4}\right) J\left(P_{i+5}\right)<0$ for all $i$. Hence the indices of $P_{j}$ and $P_{j+1}$ are different and the configuration of $A$ must be $(6)=(+,-,+,-,+,-)$.

Consider now that $A$ has configuration $(5,1)$. Assume, for instance, that the point of $A-\partial \hat{A}$ has negative index. Then $\partial \hat{A}$ has two consecutive points with positive index $P_{1}^{+}$and $P_{2}^{+}$.

First of all note that if $A$ has 3 points $P_{1}, P_{2}$, and $P_{3}$ on a straight line $L$, taking $L_{45}$ and applying the EJ formula to $L(x, y) L_{45}(x, y)$ we have that $P_{6} \in L_{45}$, so the six critical points are on two straight lines. This case is easier than the study of the general configurations of $A$ and is not treated explicitly during the proof.

Take $Q(x, y)=L_{1,2}^{++}(x, y) L_{3}^{+}(x, y)$. By the EJ formula

$$
\sum_{i=1}^{3} \frac{Q\left(P_{i}^{-}\right)}{J\left(P_{i}^{-}\right)}=0,
$$

but this is in contradiction with the fact that all $P_{i}^{-}$lies on the same connected component of $\mathbb{R}^{2}-\{Q(x, y)=0\}$ and $J\left(P_{i}^{-}\right)<0$ for $i=1,2,3$. Hence if $X \in \mathscr{Z}_{3,2}, A_{X}$ has no $(5,1)$ configuration. 
Assume now that $A$ has $(4 ; 2)$ configuration. Denote by $P_{1}, P_{2}, P_{3}$, and $P_{4}$ the points of $\partial \widehat{A}$, ordered in counterclockwise sense. Applying the EJ formula to $Q(x, y)=L_{1,2}(x, y) L_{3,4}(x, y)$ we have

$$
\frac{Q\left(P_{5}\right)}{J\left(P_{5}\right)}+\frac{Q\left(P_{6}\right)}{J\left(P_{6}\right)}=0 .
$$

Since $P_{5}$ and $P_{6}$ lie on the same connected component of $\mathbb{R}^{2}-\{Q(x, y)=0\}$, we have that $P_{5}$ and $P_{6}$ have opposite indices. Then in $\partial \widehat{A}$ there are two points with positive index $P_{1}^{+}$and $P_{2}^{+}$and two points with negative one $P_{1}^{-}$and $P_{2}^{-}$. Now we will prove that configuration $(+,-,+,-;+-)$ is not possible.

Assume that this is the case and that $P_{1}^{+}, P_{1}^{-}, P_{2}^{+}, P_{2}^{-}$are the points on $\partial \widehat{A}$ counterclockwise.

By the previous consideration we can assume that $\partial A$ is a quadrilateral. By applying the EJ formula, iteratively, to

$$
\begin{gathered}
L_{12}^{++}(x, y) L_{12}^{--}(x, y), \quad L_{12}^{++}(x, y) L_{13}^{-+}(x, y), \quad L_{12}^{++}(x, y) L_{23}^{-+}(x, y), \\
L_{12}^{--}(x, y) L_{13}^{++}(x, y), \text { and } L_{12}^{--}(x, y) L_{23}^{++}(x, y),
\end{gathered}
$$

we obtain that there is no place for $P_{3}^{-}$in $\widehat{A}$, so we have a contradiction.

Now consider that the configuration of the set $A$ is $(3 ; 3)$. If $P_{1}, P_{2}$ and $P_{3}$ are the critical points on $\partial \widehat{A}$, then by applying the EJ formula to $Q(x, y)=$ $L_{12}(x, y) L_{23}(x, y)$ and since $P_{4}, P_{5}$ have to be in the same connected component of $\mathbb{R}^{2}-\{Q(x, y)=0\}$, we obtain that they have different indices. So we can assume that $(3 ; 3)$ has configuration $(2+,-;+, 2-)$ and the proof follows.

(b) That $\left|\sum_{a \in A} i_{x}(a)\right| \in\{1,3\}$ if $X \in \mathscr{X}_{3,3}$ follows from Theorem 2.2(i).

Assume that $\sum_{a \in A} i_{x}(a)=3$. First of all we will prove that there are no critical points with index -1 in $\partial \widehat{A}$. Assume that $P_{1}^{-} \in \partial \widehat{A}$. Take $C(x, y)=L_{1}^{-}(x, y)\left(L_{23}^{--}(x, y)\right)^{2}$. Since $C\left(P_{i}^{+}\right) \geq 0, i=1, \ldots, 6$, applying the EJ formula to $C$ we get a contradiction. Hence $\#(A \cap \partial \widehat{A}) \leq 6$ and the configuration of $A$ must be $(K+; *)$ with $K \leq 6$.

Consider the case in which \# $(A \cap \partial \hat{A})=6$. Applying the EJ formula to $C(x, y)=L_{12}^{++}(x, y) L_{34}^{++}(x, y) L_{56}^{++}(x, y)$ we have a contradiction, so $\#(A \cap \partial \widehat{A}) \leq 5$. Assume that $\#(A \cap \partial \widehat{A})=5$, put $\left\{P_{1}^{+}, P_{2}^{+}, P_{3}^{+}, P_{4}^{+}, P_{5}^{+}\right\}=$ $A \cap \partial \widehat{A}$, and take a conic $Q(x, y)$ through them. Since all these points are in the boundary of a convex set, we have that the other four critical points are in the same connected component of $\mathbb{R}^{2}-\{Q(x, y)=0\}$. Assume now that there is a point $P_{6}^{+}$in the 1 st level of $A$. Taking $L_{6, K}^{+-}$where $P_{K}^{-}$is a point in $A \cap \partial \widehat{A_{1}}$ contiguous with $P_{6}^{+}$and applying the EJ formula to $Q(x, y) L_{6, K}^{+-}(x, y)$, we get a contradiction; so the configuration of $A$ is $(5+; 3-;+)$.

Step 2 (Examples). In most examples instead of explicitly giving the two polynomials $P(x, y)$ and $Q(x, y)$ such that

$$
(\dot{x}, \dot{y})=X(x, y)=(P(x, y), Q(x, y))
$$

has the configuration considered, we present a picture of the real points of $P(x, y)=0$ and $Q(x, y)=0$; see Figures 2,3 , and 4 .

Examples of configurations for systems $X \in \mathscr{X}_{3,2}$ are shown in Figure 2.

Consider now $X \in \mathscr{X}_{3,3}$ and $\sum_{a \in A} i_{x}(a)=1$. Examples of configurations $(5 ; 3 ; 1)$ and $(3 ; 6)$ are given in Figure 2 . Configuration $(4 ; 4 ; 1)$ is realized for 
the system considered in the introduction. The configuration $(4 ; 3 ; 2)$ follows from the perturbation $\dot{x}=-x\left(1+\frac{1}{2} y-\frac{3}{y} y^{2}+x^{2}\right), \dot{y}=(y+1)\left(y^{2}-y-2 x^{2}\right)-\varepsilon y$ for $\varepsilon \geq 0$ of the above system (see [Ko]). We remark that the example with configuration $(3 ; 6)$ comes from [YY], where the configuration is called type $(6 ; 3)$.

\section{REFERENCES}

[AVG] V. Arnold, A. Varchenko, and S. Goussein-Zude, Singularités des applications différentiables, Mir, Moscow, 1982.

[B] A. N. Berlinskii, On the behaviour of the integral curves of a differential equation, Izv. Vyssh. Uchebn. Zaved. Mat. 2 (1960), 3-18.

[BK] E. Brieskorn and H. Knörrer, Plane algebraic curves, Birkhäuser, Basel, Boston, and Stuttgart, 1986.

[C] W. A. Coppel, A survey of quadratic systems, J. Differential Equations 2 (1966), 293-304.

[Ch] C. Chicone and T. Jinghuang, On general properties of quadratic systems, Amer. Math. Monthly 81 (1982), 167-178.

[CL] A Cima and J. Llibre, Configurations of fans and nests of limit cycles for polynomial vector fields in the plane, J. Differential Equations 82 (1989), 71-97.

[CGM] A. Cima, A. Gasull, and F. Mañosas, On Hamiltonian planar vector fields, J. Differential Equations (to appear).

[F] W. Fulton, Algebraic curves. An introduction to algebraic geometry, Benjamin, New York, 1969.

[K] A. G. Khovanskiī, Index of a polynomial vector field, Funktsional. Anal. i Prilozhen. 13 (1979), 49-58.

[GH] P. Griffiths and J. Harris, Principles of algebraic geometry, Wiley-Interscience, New York, 1978.

[Ko] R. E. Kooij, A cubic system with $(4,-3,2)$ configuration, preprint, Univ. Tech. of Delft, 1991.

[S] A. Sestier, Note on a theorem of Berlinskil, Proc. Amer. Math. Soc. 78 (1980), 358-360.

[YY] Ye Yanqian and Ye Weiyin, A generalization of Berlinskii's theorem to cubic and quartic differential systems, preprint, 1988.

(A. Cima and F. Mañosas) Departament de Matemàtica Aplicada II, E.T.S. D'Enginyers Industrials de Terrassa, Universitat Politécnica de Catalunya, Colom, 1108222 Terrassa, Barcelona, Spain

(A. Gasull) Departament de Matemàtiques, facultat de Ciencies, Universitat autonoma de Barcelona, 08193 Bellaterra, Barcelona, Spain

E-mail address: imef2@ccuab1.uab.es 